\title{
Energy Efficient Frame Structure for Gigabit Passive Optical Networks
}

\author{
Rizwan Aslam Butt ${ }^{1}$, Sevia Mahdaliza Idrus ${ }^{2}$, Raja Zahilah Radzi ${ }^{3}$, Kashif Naseer Qureshi ${ }^{4}$ \\ ${ }^{1,2}$ Department of Electrical Engineering, University Technology Malaysia (UTM), Johor Bahru, Malaysia \\ ${ }^{3,4}$ Faculty of Computing, Universiti Teknologi Malaysia (UTM), Johor Bahru, Malaysia \\ ${ }^{1}$ Department of Electrironics Engineering, NED University of Engineering and Technology Pakistan
}

\begin{tabular}{l} 
Article Info \\
\hline Article history: \\
Received May 3, 2016 \\
Revised Oct 7, 2016 \\
Accepted Oct 21, 2016 \\
\hline
\end{tabular}

\section{Keyword:}

Energy efficiency

Frame structure

GPON

Optical access network

Passive optical network

\begin{abstract}
Increasing power consumption in information and communication access networks is one of the major cause of greenhouse gas emissions. These emissions are harmful for life on earth. Passive Optical Networks (PONs) are comparatively energy efficient network but the broadcast nature of downstream traffic leads to unnecessary frames processing by the optical network units and cause of energy wastage. Bi-PON technique has solved this problem by changing the XGPON / GPON frame structure into an interleaved pattern. However, this structure also requires additional hardware changings at the optical network units. In this paper, we have tried to achieve the same objective by making a few changings in the GPON frame structure without modifying the existing hardware structure. The simulation results show that $25.25 \%$ processing energy of an ONU can be saved by incorporating these changes.
\end{abstract}

Copyright () 2016 Institute of Advanced Engineering and Science. All rights reserved.

\section{Corresponding Author:}

Sevia Muhammad. Idrus,

Department of Electrical Engineering,

University Technology Malaysia,

P19a, Skudai, Johor Bahru, Johor-81300, Malaysia.

Email: sevia@fke.utm.my

\section{INTRODUCTION}

Recently, Information and Communication Technologies (ICTs) based networks are emerged with all fields of life and rapidly expanding [1-2]. According to latest International Telecommunication Union (ITU-T) report of 2015, the usage of internet has rised by $46 \%$, where $10.8 \%$ increased in fixed broadband and $47 \%$ increased in mobile services subscriptions [3]. This expansion has increased the electric power generation and causing of massive emission of greenhouse gases.

Around $70 \%$ of the total power consumption of the ICTs is because of Access Network [4]. Today the most widely optical access networks are Passive Optical Network (PON) which consists of only two active components: Optical Line Terminal (OLT) and Optical Network Unit (ONU). These active components are located at the central office and customer premises respectively. Due to passive nature of active components, these are more energy efficient compared to old copper based access networks but still their power consumption is not significant [5]. The current carbon foot print of this sector is almost matching withairline industry [6]. Specifically, more than $60 \%$ power is consumed by ONU while OLT consumed aonly 7\% [7]. Therefore, most of the studies have focused on ONU power reduction [8-9].

One of the main reason of ONUs power consumption is deployment of PONs and its broadcasting nature for downstream traffic due to Time Division Multiple Access (TDMA) method for transmitting to ONUs. It has been reported that in worst case 97-99\% traffic received by an ONU is un-related and discarded after processing [10]. Although WDM PON can resolved this matter but still this technology has not been commercially adopted due to its cost and requirement of separate optical fibers for every ONU from OLT 
and more over requires Thermoelectric Cooling (TEC) [11-12]. OFDM PON is another ideal candidate for future energy efficient PONs, but it suffered from synchronization problem and its complex digital signal processing operations like FFT and IFFT, which require additional computational resources [13].

In order to address aforementioned problem, the existing TDM PON techniques require physical and Media Access Control (MAC) layer changings in the ONU. Therefore techniques are not considered for existing ONU designs without redesigning its hardware circuit. Therefore, in this paper, we present a pure MAC layer based technique for GPON network and can be apply in existing ONU designs by only upgrading the ONU firmware. This technique also efficient for future ONU designs.

The rest of the paper is organized as follows: Section II discusses the related work. Section III describes the existing GPON frame structure and its processing limitations. In Section IV, proposed method illustrates with its processing mechanism. In Section V, the simulation setup and results discuss in detail. Final section concludes the paper.

\section{RELATED WORK}

Various studies have been conducted to understand and solve the ONU power consumption issue. Broadly, these techniques are categorized into Physical and MAC layer techniques or hybrid techniques [14-16]. Physical layertechniques are based on hardware changes of ONU to make it energy efficient. On the other hand, the MAC layer techniques require only firmware changes and able to tested and adopted quickly. Under this category, different techniques are available in the literature including ONU sleep mode, Dozing, Power Shedding and Awake Mode. Sleep mode is the most popular technique [16-18]. The basic idea in these techniques is to turn off the optical transmitter, receiver, CDR, and backend digital circuit completely or partially based on traffic conditions for certain time. The drawback of sleep mode techniques is its usage only in low volumes traffic situations. For heavy or moderate traffic volumes, sleep modes are not feasible due to deep sleep modes have severe impacts on Quality of Services (QoS) parameters such asdelay and jitter. A special case of sleep mode is dozing which is partial sleep. The more effective technique is a combination of sleep and doze modes like cooperative sleep and watchful sleep mode [19-20].

The idea of using a slotted mechanism for downstream has been described in but it limits the system throughput [21]. The concept of saving processing power by avoiding un-related frame processing was first proposed in [6]. A bit interleaved frame structure was introduced as Bi-PON. In this technique, an ONU only reads its own bits in PLOAM, BW MAP and GEM sections and thus saves significant processing power. Up to $30 \%$ processing power saving has been demonstrated using Bi-PON [22]. The drawback of this technique is that, it requires additional hardware changes in the ONU and requires new ONU designs and new investment.

According to Cheng and Effenberger [23], the idea of keeping ONUs sleep and only awake when their traffic arrives has described. This technique requires complex pattern recognition algorithms at OLT to predict the future arrivals and calculate awake timings for the ONUs without QoS degradations.

This work is inspired by the idea of Bi-PON and proposes a new GPON frame structure for reducing un-related processing. This idea saves significant processing energy by only making minor changes in current GPON frame structure without impacting its basic functions and services unlike the existing Bi-PON method of Bi-PON. The proposed Energy Efficient PON (EGPON) solution described in the following sections.

\section{EXISTING METHOD AND ITS LIMITATIONS}

Existing GPON network uses the frame structure as shows in Figure 1. It comprises of four main sections namely; synchronization and clock (IDENT), Physical Layer Operation and Maintenance Downstream (PLOAMd), Bandwidth (BW) MAP and GEM Payload. IDENT carries the synchronization information and OLT clock count. The PLOAMd contains instruction messages from OLT. BW-MAP contains the time slot assignments for the upstream transmissions and the GEM section contains the payload mapped to Allocation IDs (ALLOC-ID), assigned to ONUs during initialization.

Since GPON is a synchronous network, an ONU in this network receives 8000 frames per second and it is possible that none of the frames have a PLOAM or data message destined for it and all the frames are processed and discarded by it. Therefore, when it is idle, as suggested by BI-PON proposal, GPON power consumption can be significanclty reduced if only relevant downstream frames are processed by an ONU. However, the exisiting frame structure forces ONUs to process every frame. Therefore, it is necessary to change this frame structure. To understand this problem, we first explain the existing frame structure and its processing mechanism.

In this frame structure an ONU MAC processor as shows in Figure 2, has to process IDENT, PLOAM, BW MAP and GEM sections for extract OLT clock, Operation and Management (OAM) 
instructions from OLT, Upstream bandwidth assignment for upstream transmission and data from each section. From the IDENT section, it extracts OLT clock. From PLOAM section, BW MAP, and GEM sections, it extracts OAM messages, upstream bandwidth assignments, and data encapsulation in Transmission Containers (TCONTS) respectively. A TCONT is payload mapped to a specific Allocation Identifier (ALLOC-ID) of the ONU. In the BW Map and GEM sections, if the ALLOC-ID doesn't belong to the ONU then that Allocation or TCONT is discarded after processing. Therefore, if an ONU can know before processing about the Alloc-IDs included in the BW Map and GeM sections then an ONU can discard these sections without processing, if its Alloc-IDs are not included in this sections. The detailed processing steps are involved in parsing the current frame structure are shows in Figure 3, with the help of flow chart. The existing frame structure includes PLEND sections twice to make sure that if one section is spoiled due to bit errors, the other can be used. This section constrains very important field of BLEN that describes the length of the BW MAP section.

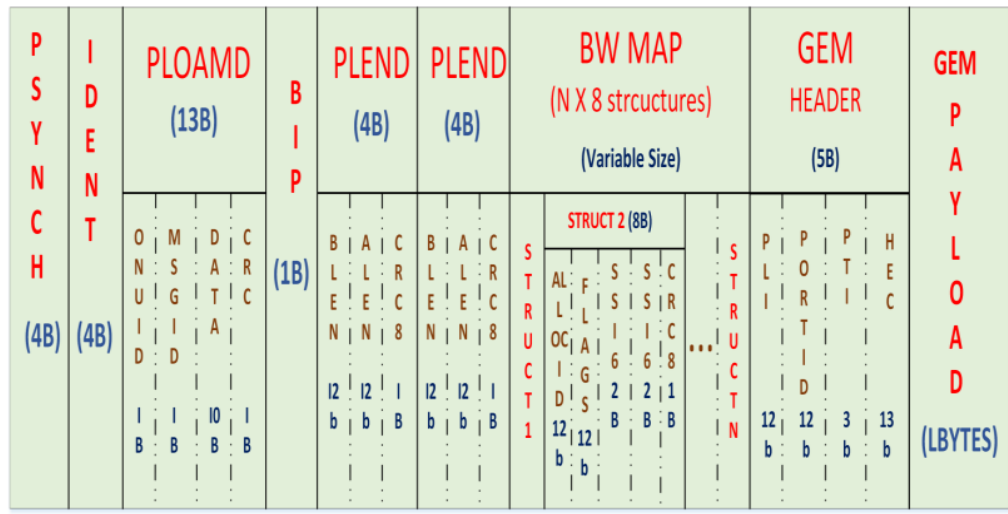

Figure 1. GPON Frame Structure

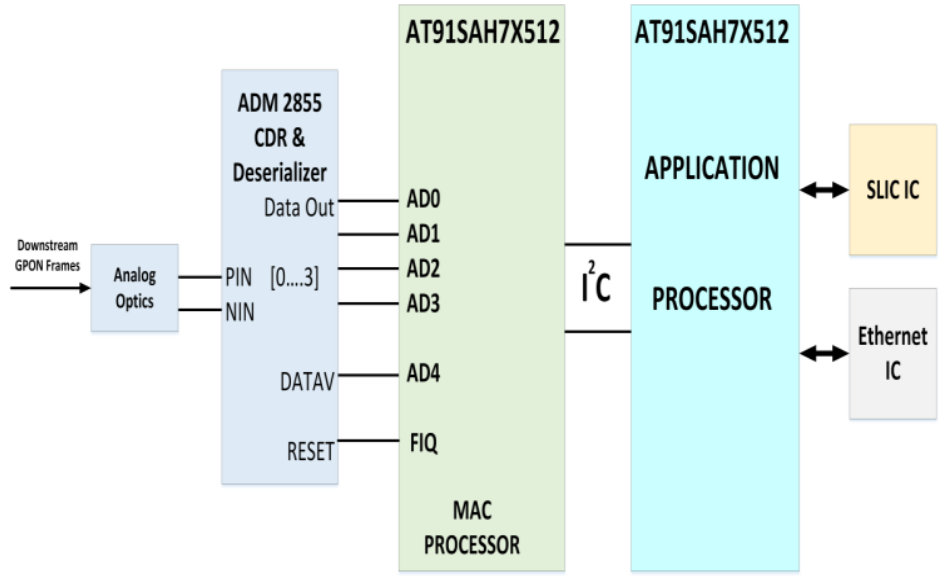

Figure 2. Downstream Frame and Processing Unit of a GPON ONU 


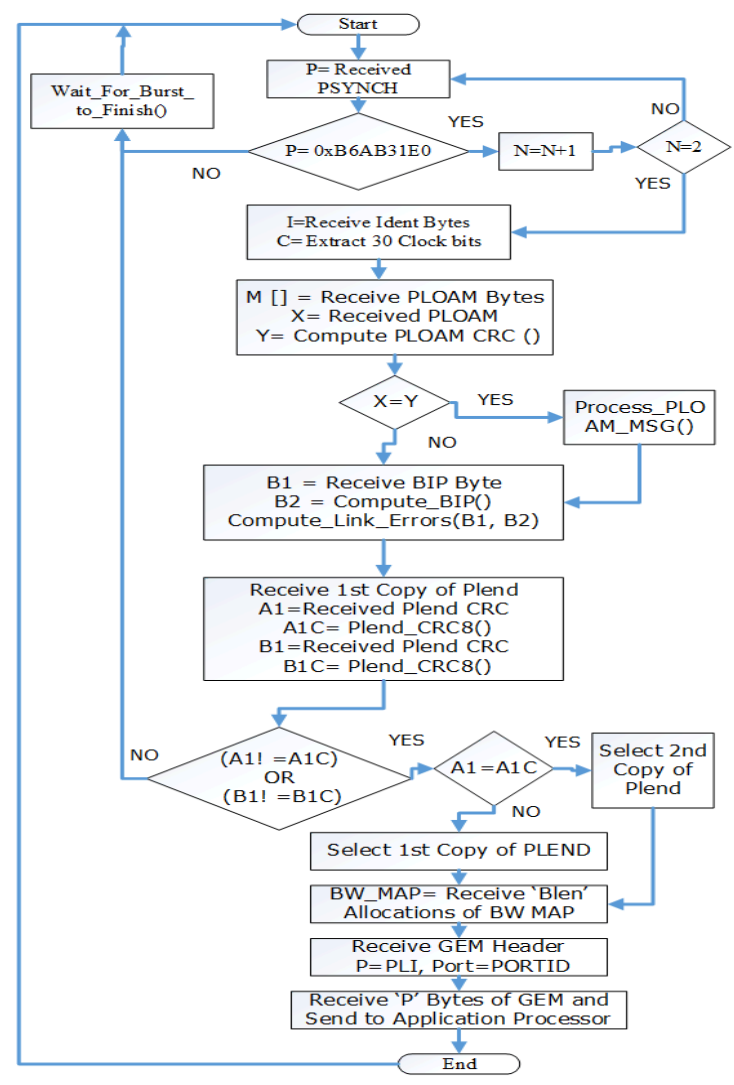

Figure 3. GPON Frame Processing by an ONU

\section{PROPOSED METHOD}

The limitation of the existing frame structure is that by using it' ONU cannot determine the relevancy of the frame unless it completely processe it. Therefore, a few frame structure for a GPON network with few changes is proposed as shows in Figure 4. These changes enable an ONU to determine the relevancy of the received frame at early stage before processing the BW MAP and GEM sections and thus save significant processing energy. The new frame structure terms as Energy Efficient GPON (EGPON).

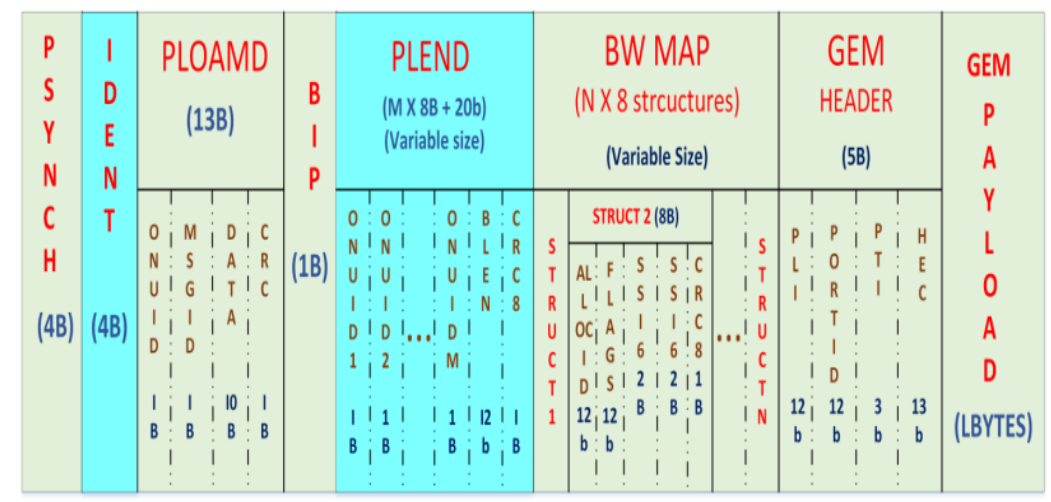

Figure 4. EGPON Frame Structure

The first change made is in the IDENT section. An existing unused bit termed as reserve bit ' $r$ ' in the IDENT section, it is renamed as PLOAM (P) to indicate the presence of the PLOAM message inside the immediately following section. So by inspecting this bit an ONU can skip processing of the PLOAMd section and save significant processing energy, if there is no PLOAM message from the OLT. 
Similarly a single GPON frame could have a maximum of 4096 upstream timeslot assignments by the OLT in the BW MAP and the same number of TCONTs in the GEM section in a single frame and a total of 32768000 per second. If an ONU is idle then it will process all these frames and simply discard as being un-related, resulting in a significant processing energy wastage. In order toaddress this problem, we recommend to add a list of ONU-IDs for all ONUs and having data or upstream time slot assignments in the current frame in the PLEND section. Now an ONU can check its ONU-ID in this list and decide whether to process further or just discard the next un-related sections. We also eliminate ATM Length (ALEN) field as it is now deprecated and not used by GPON. Moreover instead of using PLEND twice in the GPON frame structure, it is only used once in EGPON according to our analysis. CRC-8 filed can provide enough integrity for this section. We do not make any other changes in the GPON frame structure except these two.

\subsection{EGPON Frame Processing}

A Processing of EGPON frame is similar to GPON except it has two additional decision stages as shows in Figure 5.

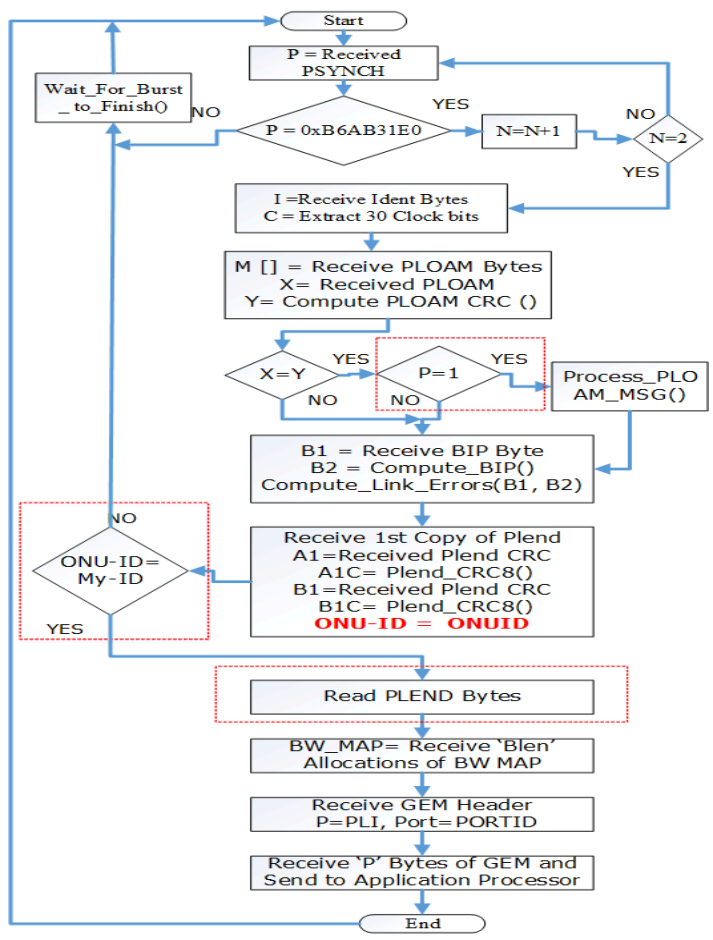

Figure 5. EGPON Frame Processing

The changes made are highlighted with a dotted rectangular box. In the first decision stage, if bit 'P' is zero then we can skip the PLOAM processing function and save PLOAM processing energy EPLOAM. OLT only makes ' $\mathrm{P}=1$ ' if there is a PLOAM message in the frame. Similarly in the second decision stage, an ONU checks its ONU-ID in the list added by the OLT in the PLEND section and discards rest of the frame if its ONU-ID is not listed there. There could be three types of frames being received by an ONU. We define type "A" frames as completely having both PLOAM and GEM as well as BW MAP sections destined for ONU. If only GEM and BW MAP section is related then these are in type "B" frames else the frames belong to type "C" which are un-related frames. It should be noted that, in most of the cases an ONU has upstream bandwidth assignment in BW MAP only when there is a relevant TCONT GEM section. So we assume either these sections are related to an ONU or both are unrelated. If EPL, EID and EBW_GEM, represent the processing energies of PLEND, IDENT and both GEM and BW MAP sections then we can write,

$$
\begin{aligned}
& E_{G P O N_{-} A}=E_{G P O N_{-} B}=E_{G P O N_{-} C}=E_{E G P O N_{-} A} \\
& E_{E G P O N_{-} A}=E_{I D}+E_{P L O A M}+E_{B W_{-} G E M}+E_{P L}
\end{aligned}
$$




$$
\begin{aligned}
& E_{E G P O N_{-} B}=E_{I D}+E_{B W_{-} G E M}+E_{P L} \\
& E_{E G P O N_{-} C}=E_{I D}+E_{P L}
\end{aligned}
$$

where the subscripts A, B and C represent the frame processing energies by ONU in case of "A", "B" and "C" which are types of GPON and EGPON frames.

\section{RESULTS AND ANALYSIS}

In order to compare the performance of EGPON with GPON frame structure, the MAC layer processor codes were written using ARM7TDMI assembly language and were compiled and simulated using Keil uVision 5.15 environment. In order to quantize the execution energy of both codes, the instruction level energy model for ARM7TDMI available in [24]. The instructions used in our codes with their execution energies in nano joules (nJ) are shows in Table 1. Only the execution energies of MAC layer processor is computed as the energy consumed by application processor and same for both GPON and EGPON frames.

Table 1. Instruction Execution Energies for ARM7TDMI Processor

\begin{tabular}{cccc}
\hline Energy $(\mathrm{nJ})$ & Instruction & Instruction & Energy (nJ) \\
\hline ADD & 0.89 & CMP & 0.978 \\
RSB & 1.153 & EOR & 1.167 \\
BIC & 1.049 & CMP & 1.143 \\
AND & 1.178 & EOR & 1.343 \\
Shift & 0.288 & CMP & 1.94 \\
B & 0.79 & EOR & 3.593 \\
BL & 6.023 & CMP & 1.284 \\
\hline
\end{tabular}

The processing logic and steps for parsing and analyzing GPON and EGPON frames are described with the help of flow charts showed in Figure 3 and Figure 4 respectively. The frame processing energies is computed for each of the three types of frames " $\mathrm{A}$ ", "B" and " $\mathrm{C}$ " using equation 1-4, for both GPON and EGPON frames asshowsin Figure 6.

It is clear evident, that in the case of type "A" frames performance of EGPON which required a little bit more processing power but in case of type "C" frames EGPON provided an energy saving of about 700nJ which amounts issaving $25.25 \%$ compared to GPON computed using Equation (5).

$$
P E=\frac{E_{E G P O N_{-} C}-E_{G P O N_{-} C}}{E_{G P O N_{-} C}} X 100
$$

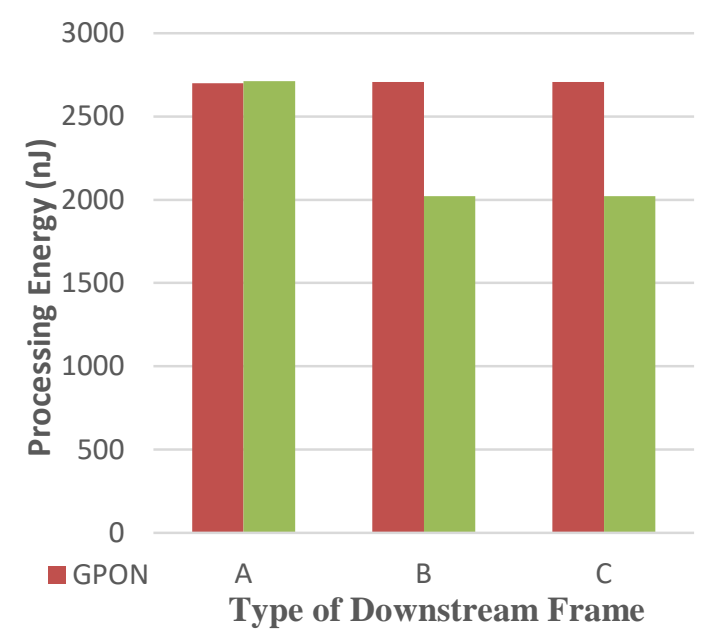

Figure 6. EGPON Frame Processing

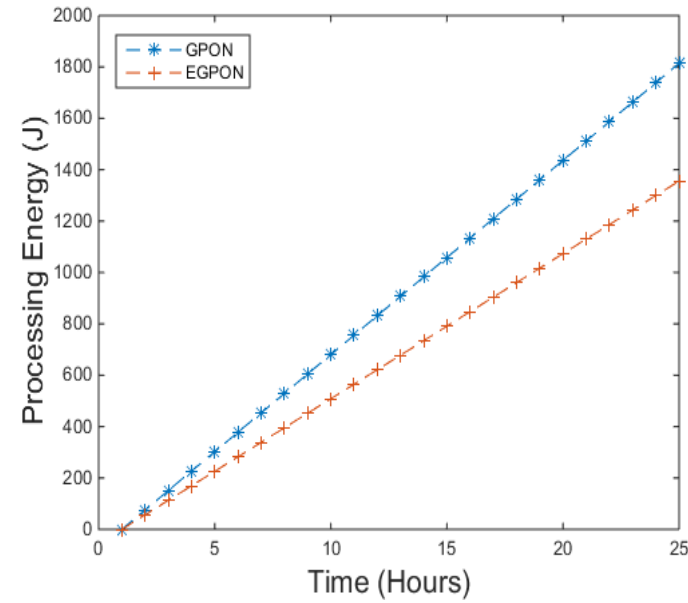

Figure 7. EGPON Frame Processing 
As mentioned by Suvakovic, et al., [6], only 3\% of the traffic reaching an ONU is related. Therefore, in one hour there is $\left[2.7936 \times 10 \rrbracket^{\wedge} 7\right.$ un-related frames reaching an ONU. Figure 7 , shows the processing energy consumption of GPON and EGPON frames per hour basis for type "C" frames. A saving of 458 Joules of processing energy is achieved in 24 hours by an ONU using EGPON frame structure.

\section{CONCLUSION}

By using EGPON frame structure instead of standard GPON, 25.25\% processing energy can be achieved by an ONU due to lesser un-related processing of downstream frames. Moreover, the suggested changes neither change the basic structure of GPON without impact on protocol functions and services. The proposed changes actually add more information to the frame structure to enable an ONU to decide the relevancy of the frame at an early stage and discard it without processing if it is un-related. The proposed solution doesn't require any additional hardware changes in the ONU andcan be applied to currently deployed GPON network by only upgrading the ONU firmware.

\section{ACKNOWLEDGEMENTS}

This work was supported by Ministry of Education Malaysia and the administration of University Technology Malaysia (UTM) for the project financial support through FRGS Fund vote number 4F565.

\section{REFERENCES}

[1] K. N. Qureshi, et al., "Road Aware Geographical Routing Protocol Coupled with Distance, Direction and Traffic Density Metrics for Urban Vehicular Ad Hoc Networks," Wireless Personal Communications, pp. 1-20.

[2] K. N. Qureshi, et al., "Geographical Forwarding Methods in Vehicular Ad hoc Networks," International Journal of Electrical and Computer Engineering, vol. 5, 2015.

[3] N. S. C. I. S. Center, "Facts and figures at a glance," University of Alabama: Birmingham, AL, USA, 2015.

[4] P. Chowdhury, et al., "Building a green wireless-optical broadband access network (WOBAN)," Journal of Lightwave Technology, vol. 28, pp. 2219-2229, 2010.

[5] S. S. Newaz, et al., "Adaptive delay-aware energy efficient TDM-PON," Computer Networks, vol. 57, pp. 15771596, 2013.

[6] D. Suvakovic, et al., "A low-energy rate-adaptive bit-interleaved passive optical network," IEEE Journal on Selected Areas in Communications, vol. 32, pp. 1552-1565, 2014.

[7] J. I. Kani, "Power saving techniques and mechanisms for optical access networks systems," Journal of Lightwave Technology, vol. 31, pp. 563-570, 2013.

[8] S. W. Wong, et al., "Sleep mode for energy saving PONs: advantages and drawbacks," in 2009 IEEE Globecom Workshops, pp. 1-6, 2009.

[9] D. P. Van, et al., "Energy-saving framework for passive optical networks with ONU sleep/doze mode," Optics express, vol. 23, pp. A1-A14, 2015.

[10] D. Suvakovic, et al., "Low energy bit-interleaving downstream protocol for passive optical networks," in Online Conference on Green Communications (GreenCom), 2012 IEEE, pp. 26-31, 2012.

[11] Y. Qi, et al., "Design and performance analysis of $2 \times 10 \mathrm{~GB} / \mathrm{S}$ bidirectional green WDM-PON based on circulator," in Broadband Network and Multimedia Technology (IC-BNMT), 2011 4th IEEE International Conference on, pp. 56-60, 2011.

[12] B. Quan, et al., "Dynamic Routing and Resource Assignment Algorithm In sloted optical Networks," Indonesian Journal of Electrical Engineering and Computer Science, vol. 11, pp. 1813-1821, 2013.

[13] Y. Du and J. Zhang, "The Performance of Synchronization Algorithm in Real-time OFDM-PON System," Indonesian Journal of Electrical Engineering and Computer Science, vol. 10, pp. 1785-1794, 2012.

[14] E. Wong, et al., "Energy-efficiency of optical network units with vertical-cavity surface-emitting lasers," Optics express, vol. 20, pp. 14960-14970, 2012.

[15] R. Kubo, et al., "Sleep and adaptive link rate control for power saving in 10G-EPON systems," in Global Telecommunications Conference, 2009. GLOBECOM 2009. IEEE, pp. 1-6, 2009.

[16] J. Zhang and N. Ansari, "Toward energy-efficient 1G-EPON and 10G-EPON with sleep-aware MAC control and scheduling," IEEE Communications Magazine, vol. 49, pp. s33-s38, 2011.

[17] L. Shi, et al., "Energy-efficient PON with sleep-mode ONU: progress, challenges, and solutions," IEEE Network, vol. 26, pp. 36-41, 2012.

[18] H. Bang, et al., "Determination of sleep period for cyclic sleep mode in XG-PON power management," IEEE Communications Letters, vol. 16, pp. 98-100, 2012.

[19] D. N. V. Fernando, et al., "Cooperative cyclic sleep and doze mode selection for NG-PONs," in 2014 16th International Conference on Transparent Optical Networks (ICTON), pp. 1-4, 2014.

[20] R. O. Hirafuji, et al., "The watchful sleep mode: a new standard for energy efficiency in future access networks," IEEE Communications Magazine, vol. 53, pp. 150-157, 2015.

[21] S. S. Lee and A. Chen, "Design and analysis of a novel energy efficient ethernet passive optical network," in Networks (ICN), 2010 Ninth International Conference on, pp. 6-9, 2010. 
[22] H. Chow, et al., "Demonstration of low-power bit-interleaving TDM PON," in European Conference and Exhibition on Optical Communication, 2012.

[23] N. Cheng and F. Effenberger, "Automatic ONU wavelength control in TWDM PONs," in Optical Fiber Communication Conference, 2014.

[24] M. Bazzaz, et al., "An accurate instruction-level energy estimation model and tool for embedded systems," IEEE Transactions on Instrumentation and Measurement, vol. 62, pp. 1927-1934, 2013. 